\title{
Characterization of Focal Liver Lesions using CEUS and MRI with Liver-Specific Contrast Media: Experience of a Single Radiologic Center
}

\section{Charakterisierung von fokalen Leberläsionen mit CEUS und MRT mit leberspezifischem Kontrastmittel: Erfahrungen eines radiologischen Zentrums}

Authors

Lukas Philipp Beyer, Florian Wassermann, Benedikt Pregler, Katharina Michalik, Janine Rennert, Isabel Wiesinger, Christian Stroszczynski, Philipp Wiggermann, Ernst Michael Jung

\section{Affiliation}

Department of Radiology, University Hospital Regensburg, Germany

Key words

CEUS, MRI, HCC, liver-specific contrast media, focal liver lesions

received 15.01.2016

accepted 28.02.2017

Bibliography

DOI http://dx.doi.org/10.1055/s-0043-105264

Published online: November 6, 2017 | Ultraschall in Med 2017; 38: 619-625 @ Georg Thieme Verlag KG Stuttgart . New York

ISSN 0172-4614

Correspondence

Dr. Lukas Philipp Beyer

Department of Radiology, University Hospital Regensburg

Franz-Josef-Strauß-Allee 11

93053 Regensburg

Germany

Tel.: ++ 49/941/9447401

Fax: ++ 49/9 41/9447409

lukas@lukasbeyer.com

\section{ABSTRACT}

Aim The purpose of this study was to compare contrast-enhanced ultrasound (CEUS), magnetic resonance imaging (MRI) using liver-specific contrast agent and a combination of both for the characterization of focal liver lesions (FLL).

Methods 83 patients with both benign and malignant liver lesions were examined using CEUS and MRI after the intravenous administration of liver-specific contrast media. All patients had inconclusive results from prior imaging examinations. Histopathological specimens could be obtained in 53 patients. Ultrasound was performed using a multi-frequency curved probe $(1-6 \mathrm{MHz})$ after the injection of $1-2.4 \mathrm{ml}$ ultrasound contrast media. The sensitivity, specificity, positive predictive value and negative predictive value of CEUS, MRI and a combination of both (CEUS + MRI) were compared.

Results The sensitivity, specificity, positive and negative predictive values regarding lesion classification were $90.9 \%$, $70.6 \%, 92.3 \%$ and $66.6 \%$, respectively, for CEUS; $90.9 \%$, $82.4 \%, 95.2 \%$ and $70.0 \%$, respectively, for MRI; and $96.9 \%$, $70.6 \%, 92.7 \%$ and $85.7 \%$ respectively, for CEUS + MRI. There were no statistically significant differences. 6 malignant lesions were missed using CEUS or MRI alone (false negatives). The use of both modalities combined reduced the false-negative results to 2 .

Conclusion CEUS and MRI with liver-specific contrast media are very reliable and of equal informative value in the characterization of focal liver lesions. The number of false-negative results can be decreased using a combination of the two methods.

\section{ZUSAMMENFASSUNG}

Ziel Vergleich der Aussagekraft von CEUS und MRT mit leberspezifischem Kontrastmittel sowie einer Kombination aus beiden Modalitäten zur Charakterisierung fokaler Leberläsionen (FLL).

Methoden Insgesamt wurden 83 Patienten mit benignen und malignen Leberläsionen mittels CEUS und MRT mit leberspezifischem Kontrastmittel untersucht, nach vorangegangener Schnittbilddiagnostik ohne eindeutiges Ergebnis. Eine histo-pathologische Sicherung der Läsionen erfolgte bei 53 Patienten. Die Sensitivität und Spezifität sowie der positiv und negativ prädiktive Wert von CEUS, MRT mit leberspezifischem Kontrastmittel und der Kombination aus beiden Bildgebungen (CEUS + MRT) wurden verglichen. Die Ultraschalluntersuchungen wurden mit einer Multifrequenz Konvexsonde $(1-6 \mathrm{MHz})$ nach Applikation von 1-2,4 ml Ultraschallkontrastmittel durchgeführt.

Ergebnisse Die Sensitivität, die Spezifität, der positiv und der negativ prädiktive Wert bezogen auf die Dignität der untersuchten Läsionen waren für CEUS 90,9\%, 70,6\%, 92,3\% und $66,6 \%$, für MRT 90,9\%, 82,4\%, 95,2\% und 70,0\% und für 
CEUS + MRT 96,9\%, 70,6\%, 92,7\% und 85,7\%. Die Unterschiede waren nicht statistisch signifikant. Bei Beschränkung auf nur eine bildgebende Modalität (CEUS oder MRT) wurden jeweils 6 maligne Läsionen nicht erkannt (falsch negativ). Eine Kombination aus beiden Bildgebungen (CEUS + MRT) konnte die Anzahl der falsch negativen Ergebnisse auf 2 reduzieren.
Zusammenfassung Sowohl CEUS als auch MRT mit leberspezifischem Kontrastmittel haben bei der Charakterisierung von fokalen Leberläsionen einen hohen diagnostischen Stellenwert. Die Kombination aus beiden Bildgebungen führt zu einer Verringerung falsch negativer Ergebnisse.

\section{Introduction}

The most important role of diagnostic imaging of focal liver lesions (FLL) is the characterization of lesions regarding malignancy. The differentiation between benign (e. g. hemangiomas, adenomas and focal nodular hyperplasia) and malignant (e.g. hepatocellular carcinomas [HCC], cholangiocellular carcinomas [CCC] and metastases) lesions determines the individual prognosis and the subsequent treatment. Benign asymptomatic FLLs do not typically require additional treatment. For malignant lesions, a number of treatment options, such as local ablative methods, resection, and chemotherapy, are possible depending on the exact entity.

Most focal liver lesions are detected in a non-contrast-enhanced ultrasound examination. However, sometimes it remains unclear whether the lesion is benign or malignant [1]. In addition to contrast-enhanced computed tomography and contrast-enhanced magnetic resonance imaging, contrast-enhanced ultrasound (CEUS) has become an established alternative for quick characterization of lesions in recent years [2, 3]. In CEUS, the vascularization and even the micro-vascularization of liver lesions can be shown in real time with the help of highly echogenic, strictly intravascular sulfur hexafluoride microbubbles (SonoVue ${ }^{\circledR}$, Bracco, Milan, Italy). This allows precise characterization of the lesion [4] and specific vascularization patterns can be observed. CEUS therefore (provided the examiner is experienced) often provides the solution in cases of unclear CT or MRI findings, especially in small lesions [5]. Furthermore, new liver-specific intracellular MRI contrast agents seem to increase sensitivity especially for small lesions [6-8].

The aim of this study was to compare CEUS and MRI using liverspecific contrast agents as well as a combination of both techniques in the characterization of focal liver lesions.

\section{Materials and Methods}

\section{Study Population}

For this retrospective study, we reviewed all contrast-enhanced ultrasound and contrast-enhanced MRI examinations of the liver in the department of diagnostic radiology and the interdisciplinary ultrasound center over a period of 2 years between 01/2011 and $01 / 2013$. All patients who underwent both examinations, i. e., CEUS and MRI using liver-specific contrast agent Resovist ${ }^{\circledR}$ (Ferucarbotran) or Primovist ${ }^{\circledR}$ (Gd-EOB-DTPA disodium salt), within 14 days without any intermediate therapy were included. Patients with contraindications for either SonoVue ${ }^{\circledR}$ or liver- specific MRI contrast agent (Resovist ${ }^{\circledR}$ or Primovist ${ }^{\circledR}$ ) were excluded. Written informed consent for CEUS and MRI was obtained in all cases. Our institutional review board did not require approval for this retrospective study.

In total, 895 patients underwent contrast-enhanced MRI of the liver and 729 patients received CEUS. The number of cases in which both imaging methods were performed in a complementary manner and within 14 days was 125.83 out of these 125 patients received liver-specific contrast agent (Resovist ${ }^{\circledR} n=32$ and Primovist ${ }^{\circledR} n=51$ ) and were therefore included in our study ( $>$ Table 1).

In cases with more than one lesion, a main lesion per imaging method was identified for every case. Therefore, if multiple suspicious lesions were found in a single patient, only one "target lesion" was included in the study. If a possibly malignant mass and a benign tumor, for which the benign status had already been definitively determined using B-mode and color-coded Doppler ultrasound, were found in a single patient, only the presumably malignant tumor was further examined.

Patients were referred to our radiology department for further diagnostic imaging either by their GP or by different departments within the hospital, if they had already been examined by other imaging methods (conventional ultrasound, CT or MRI with nonliver specific contrast media) and the FLL still remained unclear.

\section{Contrast-Enhanced Ultrasound (CEUS)}

CEUS was performed with a high-end ultrasound machine (LOGIQ E9, GE, Chalfont, UK) by an experienced examiner using a $1-6 \mathrm{MHz}$ curved probe. First, the whole liver was scanned in B-mode and the lesion was identified. CCDS and power Doppler were then performed. Afterwards, a bolus of $1-2.4 \mathrm{ml}$ contrast media was applied followed by a bolus of $\mathrm{NaCl}$.

SonoVue ${ }^{\circledR}$ (Bracco, Milan, Italy) was used in all examinations. SonoVue ${ }^{\circledR}$ is a second-generation contrast agent. The sulfur hexafluoride microbubbles have a phospholipid coating, which makes them soluble in blood. They have an average diameter of $2-10 \mu \mathrm{m}$ and thus pass easily through the capillary bed. The boundary surface between sulfur hexafluoride gas and blood reflects the ultrasound waves, resulting in an increase in the contrast between tissue and blood [9].

SonoVue ${ }^{\circledR}$ is a purely intravascular contrast agent unlike liverspecific MRI contrast agent that diffuses through membranes and is therefore considered an intracellular contrast agent [8].

The following criteria were used to characterize liver lesions:

1. The contrast of a lesion in relation to the surrounding liver parenchyma (hypo-, iso-, hyper-enhanced) in the arterial phase (starting $10-20 \mathrm{sec}$ after injection), the portal venous 
- Table 1 Patient characteristics.

\section{characteristic}

total number of patients

mean age

age range

sex

liver cirrhosis
83

59.8 years

$18-78$ years

24 (28.9\%) female

$59(71.1 \%)$ male

$44(53 \%)$ phase (starting 30 - $45 \mathrm{sec}$ after injection) and the late phase (>120 sec up to 5 min. after injection);

2. The distribution of the contrast agent in the lesion (central, peripheral); and

3. The specific vascularization pattern (rim enhancement, wheel spoke sign).

Contrast agent behavior in the late phase is particularly important with respect to determining whether a lesion is malignant or benign - benign lesions are usually iso- or hyper-enhanced, while malignant lesions are hypo-enhanced, when showing so-called wash-out.

Overall, malignant liver lesions (both primary and secondary) show wash-out in the late phase, sometimes starting in the portal venous phase, while benign lesions tend to have increased uptake of contrast media and no wash-out [9].

\section{Magnetic Resonance Imaging (MRI)}

All examinations were performed on a 1.5T MRI unit (Siemens Avanto, Siemens AG Healthcare, Erlangen, Germany). The pre-contrast protocol consisted of the following sequences: axial T2-weighted HASTE, T2-weighted BLADE, T1-weighted FLASH in- and opposed-phase and T1-weighted VIBE $3 \mathrm{D}$. Dynamic axial T1-weighted VIBE scans were obtained 20, 40 and $120 \mathrm{~s}$ after the injection of either Gd-EOB-DTPA (25 mmol/ $\mathrm{kg}$ ) or Gd-DTPA $(0.2 \mathrm{mmol} / \mathrm{kg})$. The hepatobiliary phase sequences were acquired 20 minutes after injection of Gd-EOB-DTPA (axial T1-weighted VIBE) or $1.4 \mathrm{~mL}$ Ferucarbotran (axial T2-star-weighted Flash 2D and T2-weighted TSE FS).

The following examination sequences were acquired: Axial T2 HASTE and T2 BLADE, axial diffusion/ADC, axial T1 VIBE3 D native, arterial, portal venous and late phase as well as coronal T1 VIBE3 D in the late phase. The images were interpreted by two radiologists experienced in abdominal imaging according to known criteria $[10,11]$.

Indications for the application of liver-specific contrast media were:

1. Patients with liver cirrhosis;

2. Diagnosis and surgical planning of patients with liver metastasis, because even small lesions $(<0.2 \mathrm{~cm})$ can be detected using liver-specific contrast-media;

3. Differentiation between FNH and adenoma [12].
- Table 2 Final classification of all examined FLLs. Other includes entities such as regenerative nodules and an angiomyolipoma.

\begin{tabular}{|l|r|r|}
\hline entity & $\mathbf{n}$ & histopathology \\
\hline hemangioma & 3 & $1(33 \%)$ \\
\hline adenoma & 4 & $3(75 \%)$ \\
\hline cholangiocellular carcinoma & 6 & $5(83 \%)$ \\
\hline metastasis & 17 & $13(76 \%)$ \\
\hline hepatocellular carcinoma & 42 & $26(62 \%)$ \\
\hline other & 11 & $5(45 \%)$ \\
\hline
\end{tabular}

Liver-specific contrast media is taken up by the healthy liver cells and is eliminated through the kidneys and the liver almost equally $[12,13]$.

\section{Diagnostic verification}

When combining CEUS and MRI (CEUS + MRI), an FLL was classified as malignant if at least one of the two imaging modalities, CEUS or MRI, suspected malignancy. The final lesion classification was determined by combining all information from imaging, clinical information (i. e., cirrhosis, any known cancer) and where available from histopathological analyses of the biopsy $(n=16)$ or surgical resection specimen $(\mathrm{n}=37)$. In cases without histopathology, the lesions were evaluated for changes in size and/or appearance with follow-up imaging and clinical examinations for a period of at least 12 months. - Table 2 shows an overview of the final classification of all examined FLLs.

\section{Statistics}

All FLLs were classified as benign, malignant, or indeterminate. The concordance and discordance of the characterization regarding malignancy and tumor classification (e. g. HCC, metastasis) were calculated for all patients. The sensitivity, specificity, and positive and negative predictive values were calculated for all lesions

\section{Results}

Of the 83 lesions, 66 (79.5\%) were malignant and 17 (20.5\%) were benign. In 8 (47.1\%) of the 17 benign tumors and 45 (68.2\%) of the 66 malignant tumors, the diagnosis was histopathologically proven. In the remaining cases, further imaging and clinical follow-up verified the diagnosis.

Using both CEUS and MRI, concordant results regarding the evaluation of malignancy were seen in 67 cases (80.7\%), and discordant results were seen in 6 cases (7.2\%) as shown in - Table 3. In 2 (2.4\%) out of the remaining 10 (12.0\%) cases, the lesion could not be definitively categorized in both imaging modalities. In both cases the definitive diagnosis (1 regenerative nodule, 1 angiomyolipoma) was determined via biopsy and histopathology. The other 8 (9.6\%) lesions remained unclear in either CEUS or MRI and were also further characterized by histopathology (5 HCCs, 1 regenerative nodule, $1 \mathrm{NHL}$ and 1 adenoma). 
- Table 3 Discordant cases in which the characterizations by CEUS and MRI with respect to malignancy do not correspond. Liver cirrhosis was present in all cases.

\begin{tabular}{|l|l|l|l|}
\hline definitive diagnosis & CEUS & MRI & diagnostic verification \\
\hline HCC & HCC & regenerative nodule & follow-up \\
\hline HCC & HCC & regenerative nodule & follow-up \\
\hline HCC & HCC & regenerative nodule & follow-up \\
\hline HCC ( $\bullet$ Fig. 1) & not detected & HCC & follow-up \\
\hline regenerative nodule & HCC & regenerative nodule & follow-up \\
\hline adenoma & HCC & regenerative nodule & histopathology \\
\hline
\end{tabular}
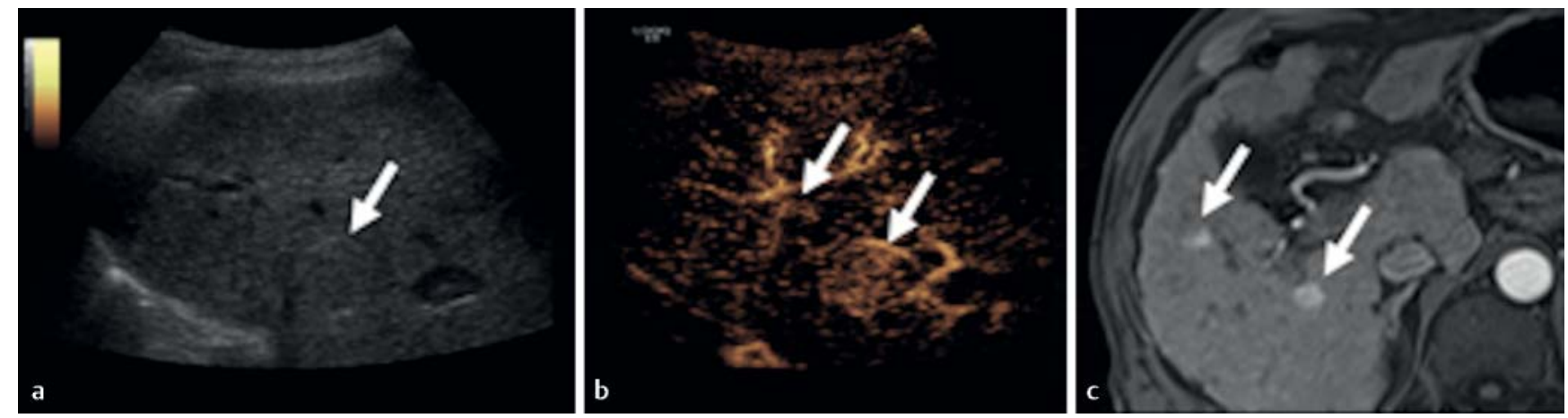

- Fig. 1 Two small HCC lesions with diameters $<2 \mathrm{~cm}$ in liver segment VI. Only the larger HCC is visible in B-mode a. Although both lesions show typical arterial hypervascularization, the smaller lesion was not detected in the initial CEUS b examination. Both lesions were detected in MRI c.

In each imaging modality, 6 out of 83 lesions were falsely classified as benign (false-negative cases). When combining both CEUS and MRI, the number of false-negative results was reduced to 2 lesions ( $\triangleright$ Table 4 ). Both false-negative lesions were cases of HCC, undetermined by CEUS. MRI falsely classified one HCC as a regenerative nodule and the other one as an arterio-venous shunt.

All of the benign lesions that were false-positive, i.e. malignant, in CEUS only were also classified as malignant in MRI $(n=5)$ : One sclerosed hemangioma with atypical arterial rim enhancement in the arterial phase during MRI and CEUS was classified as a metastasis in a patient with tonsil cancer. One adenoma with central hemorrhage was falsely classified as an $\mathrm{HCC}$ in both modalities as it showed heterogeneous arterial enhancement and hypo-enhancement in the late phase. Two hepatic abscesses showed peripheral arterial rim enhancement with wash-out in the portal venous phase and hypo-enhancement in the late phase. The abscesses were misclassified as HCC and CCC in patients with liver cirrhosis and local cholestasis, respectively. One dysplastic regenerative nodule showed arterial contrast enhancement with partial wash-out and was incorrectly classified as an HCC.

5 FLLs, proved to be benign following histopathological analyses ( 3 regenerative nodules, 1 hemangioma and 1 angiomyolipoma), were incorrectly classified as malignant by at least one imaging modality (false-positive cases). When both imaging methods categorized the lesion as benign, the diagnosis was $100 \%$ sensitive and specific.

The exact characterization of lesions regarding entity (e. g. HCC) showed concordant results in 64 cases (77.1\%) and discordant results in 8 cases $(9.6 \%)$. In 11 cases (13.3\%), the lesion was not precisely classified by at least one imaging method ( $>$ Table 5).

Overall the sensitivity, specificity, positive and negative predictive values regarding lesion characterization were $90.9 \%, 70.6 \%$, $92.3 \%$ and $66.6 \%$, respectively, for CEUS; $90.9 \%, 82.4 \%, 95.2 \%$ and $70.0 \%$, respectively, for MRI; and $96.9 \%, 70.6 \%, 92.7 \%$ and $85.7 \%$ respectively, for CEUS and MRI combined. Overall, there were no statistically significant differences between CEUS and MRI alone, and a combination of both respectively.

\section{Discussion}

Ultrasound is a common imaging modality. Abdominal ultrasound and especially ultrasound of the liver is a quick and relatively easy procedure during the daily routine. Depending on the medical history, e.g. underlying cirrhosis or any known cancer, accidental findings of FLL can be stratified towards benign or malignant lesions.

Many studies have already pointed out the advantages of CEUS compared to conventional US with regards to the characterization of focal liver lesions [14-26]. Nevertheless, CT and MRI still take 
- Table 4 Classification of all lesions in CEUS, MRI and a combination of both (CEUS + MRI) in relation to the gold standard.

\begin{tabular}{|l|l|l|l|l|}
\hline & false-negative & false-positive & true-negative & true-positive \\
\hline CEUS & 6 & 5 & 12 & 60 \\
\hline MRI & 6 & 3 & 14 & 60 \\
\hline CEUS + MRI & 2 & 5 & 12 & 64 \\
\hline
\end{tabular}

- Table 5 Classification of CEUS and MRI regarding concordance.

\begin{tabular}{|l|l|l|l|}
\hline & concordance & discordance & indeterminate \\
\hline dignity (benign/malignant) & $67(80.7 \%)$ & $6(7.2 \%)$ & $10(12.0 \%)$ \\
\hline entity (e. g. HCC, CCC) & $64(77.1 \%)$ & $8(9.6 \%)$ & $11(13.3 \%)$ \\
\hline
\end{tabular}

the dominant role in the classification of FLLs. For example, in the evaluation of HCC the American (AASLD) and European (EASL) guidelines still exclusively accept contrast-enhanced CT and/or contrast-enhanced MRI for definite diagnosis, while other guidelines recommend CEUS as the first diagnostic tool $[27,28]$ or as a second-line imaging modality in the case of inconclusive CT and MRI [29]. Recent studies have proven that CEUS can help characterize FLLs and differentiate between malignant and benign [30, 31]. Furthermore, CEUS is recommended for the diagnostic workup of patients with colorectal cancer [32]. Similar to the reporting standards of MRI and $C T$, a reporting algorithm for CEUS has been established, LI-RADS CEUS, which ought to be used for standardized reporting in liver lesions for patients at risk of HCC [33].

So far, only a few studies have evaluated CEUS as a first-line diagnostic tool in the characterization of FLLs compared to MRI. A multicenter study coordinated by the DEGUM (German Society of Ultrasound in Medicine) [34] including 269 patients concluded that liver CEUS and liver MRI were concordant in $85.9 \%$ of cases in terms of lesion entity, which is similar to the value of $80.7 \%$ calculated in our study.

Another study by D'Onofrio et al. [35] compared the diagnostic accuracy of the late phase of CEUS and the hepatobiliary phase of MRI using liver-specific contrast media in the characterization of FLLs. A total of 147 FLLs were analyzed retrospectively with a sensitivity, specificity, PPV and NPV of $90 \%, 93 \%, 97 \%$ and $80 \%$, respectively, for the late phase of CEUS, $91 \%, 93 \%, 97 \%$ and $81 \%$, respectively, for the hepatobiliary phase of MRI and $98 \%$, $98 \%, 99 \%$ and $95 \%$, respectively, for a combination of both techniques. Unlike our study, they only evaluated the late phase of CEUS and the hepatobiliary phase of MRI, especially the arterial phase. While the late phase plays an important role in the determination of the malignancy of FLLs, other imaging properties like contrast kinetics in CEUS and MRI as well as native MRI sequences like T2- and/or diffusion-weighted sequences (DWI) should also be considered. For example, studies have shown that DWI is useful in the detection of small HCCs in the cirrhotic liver as well as metastases compared to conventional contrast-enhanced imaging [36, 37].

CEUS is strictly intravasal, whereas contrast media in MRI can pass through membranes. Therefore, the contrast behavior between CEUS and MRI is different. When CEUS shows wash-out in malignant lesions, MRI might present prolonged enhancement because of leakage of contrast media into the tumor interstitium [35].

Indications for the application of liver-specific contrast media are diagnosis and staging in patients with liver cirrhosis, diagnosis and surgical planning in patients with liver metastasis, because even small lesions $(<0.2 \mathrm{~cm})$ can be detected using liver-specific contrast media, and differentiation between FNH and adenoma in cases of benign lesions [12].

All of the patients evaluated in this study only received liverspecific contrast agent. We think that our study is a valuable addition to the current literature, because previous studies have shown that liver-specific contrast agents increase the sensitivity in MRI examinations especially for small FLLs [6-8]. It is recommended by the ESGAR consensus to use liver-specific contrast media for MRI of the liver. Hence, together with the appropriate clinical information, a definite diagnosis can be made [13].

Our study showed sensitivities and specificities of $90.9 \%$ and $70.6 \%$, respectively, for CEUS and $90.9 \%$ and $82.4 \%$, respectively for MRI. We attribute the comparatively low specificity in our study to the fact that 5 benign lesions were incorrectly classified as malignant lesions by CEUS and MRI. This was partly due to poor image quality, e. g. breathing artifacts in MRI ( $>$ Fig. 2) or adiposity in CEUS as well as the atypical imaging features of the misclassified FLLs (1 adenoma, 1 sclerosed hemangioma, 2 abscesses, 1 dysplastic nodule). If the entity of a lesion remains unclear in imaging, a definitive diagnosis by biopsy - as performed in our study - should be planned. If the acquiring of a histopathological specimen is not possible, a short-term followup is essential to monitor the size of the lesion. 

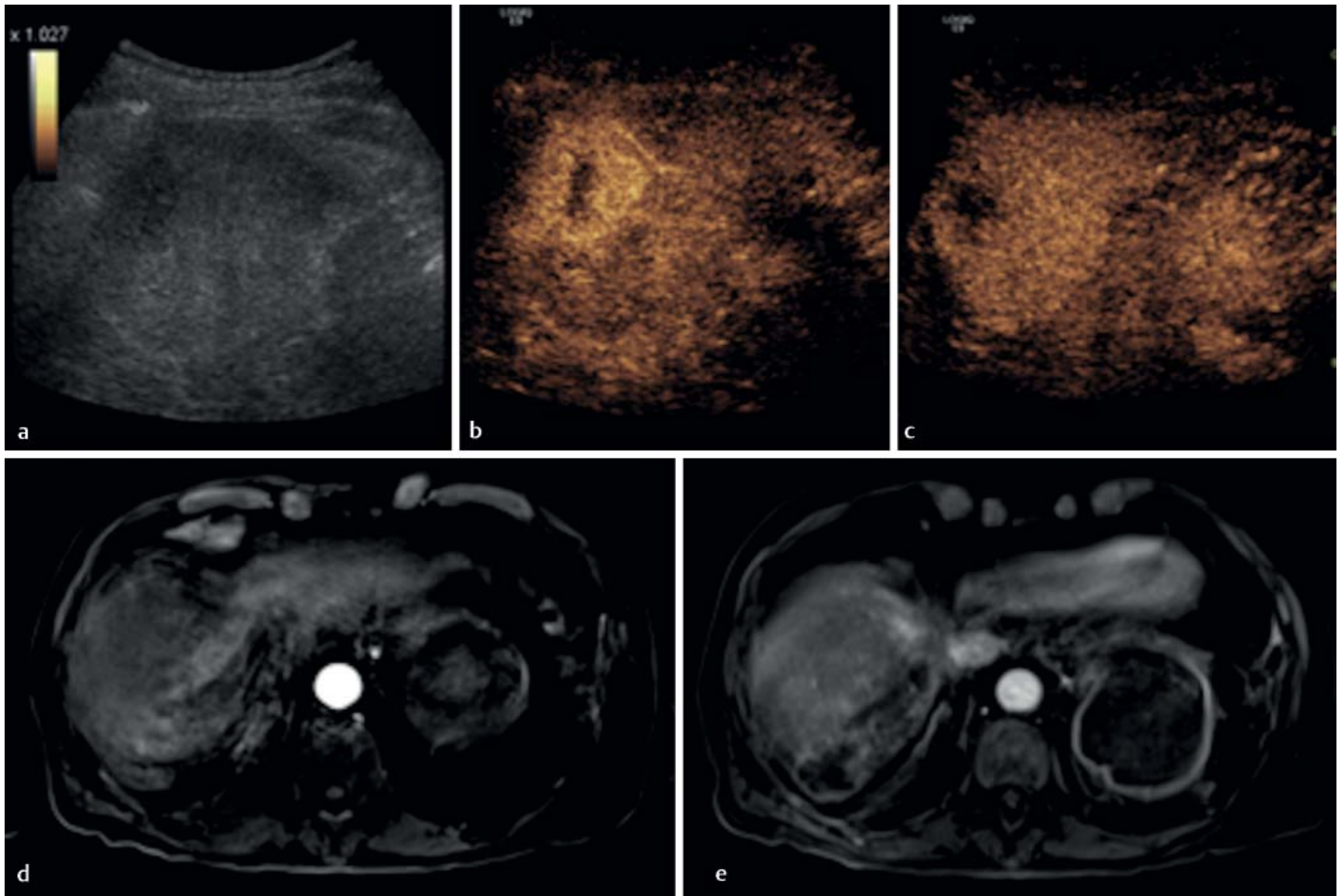

- Fig. 2 Typical contrast enhancement pattern of a singular HCC with a diameter of $3 \mathrm{~cm}$ in liver segment VII not classified in B-mode a. By optimizing the ultrasound conditions, CEUS was able to show typical arterial hypervascularization $\mathbf{b}$ with central necrosis and wash-out in the portal venous phase $\mathbf{c}$. The HCC was not detected in MRI due to heavy breathing artifacts in the arterial $\mathbf{d}$ and portal venous $\mathbf{e}$ phase.

The differentiation between regenerative nodules and HCCs in the case of liver cirrhosis is challenging. CEUS and MRI are both limited in these cases with regards to exact lesion classification. Thus, the combination of both imaging techniques allows a reduction of false-negative diagnoses (e. g., the classification of an HCC as a regenerative nodule). Therefore, we think it is important to combine the information given by both imaging modalities and the clinical input to increase diagnostic confidence. Considering the importance of exact lesion classification, we would still recommend liver biopsy in case of doubt. A combination of CEUS and MRI leads to higher expenses. Even though the cost for CEUS was estimated to be 85 EUR as compared to 175 EUR for MRI in an Italian study [38], double examination seems to be justified by the improved diagnostic accuracy.

This study is limited by its retrospective design, missing histological verification for about half of the patients, the lack of consideration of the lesion size and the different liver-specific contrast media used for MRI. Another limitation is the monocentric setup and the fact that our university hospital is a liver cancer center. This lead to a bias in the patient population with a high incidence of liver cirrhosis and increased incidence of malignant liver lesions compared to a normal patient population. The low number of benign lesions ( $\mathrm{n}=17$ ) made it impossible to draw a reliable conclusion for these cases.

In summary, CEUS and MRI of the liver using a liver-specific contrast agent are approximately equally effective imaging modalities with regard to the detection and characterization of FLLs. A combination of both imaging modalities might be able to help to reduce the number of false-negative results. Future multi-center evaluations should be performed by CEUS and MRI with liverspecific contrast agent of benign and malignant liver tumors to evaluate the final diagnostic accuracy and generalize our monocentric results.

\section{Conflict of Interest}

The authors declare that they have no conflict of interest.

\section{References}

[1] Reinhold C, Hammers L, Taylor CR et al. Characterization of focal hepatic lesions with duplex sonography: findings in 198 patients. Am J Roentgenol 1995; 164: 1131 - 1135

[2] Lencioni R, Della Pina C, Crocetti L et al. Clinical management of focal liver lesions: the key role of real-time contrast-enhanced US. Eur Radiol 2007; 17 (Suppl. 6): F73-F79 
[3] Tranquart F, Correas JM, Ladam Marcus V et al. Real-time contrast-enhanced ultrasound in the evaluation of focal liver lesions: diagnostic efficacy and economical issues from a French multicentric study. J Radiol 2009; 90: 109-122

[4] Claudon M, Dietrich CF, Choi BI et al. Guidelines and good clinical practice recommendations for contrast enhanced ultrasound (CEUS) in the liver - Update 2012. Ultraschall in Med 2013; 34: 11-29

[5] Strobel D, Bernatik T, Blank W et al. Diagnostic accuracy of CEUS in the differential diagnosis of small $(\leq 20 \mathrm{~mm})$ and subcentimetric $(\leq 10 \mathrm{~mm})$ focal liver lesions in comparison with histology. Results of the DEGUM multicenter trial. Ultraschall in Med 2011; 32: 593-597

[6] Bluemke DA, Sahani D, Amendola M et al. Efficacy and safety of MR imaging with liver-specific contrast agent: U.S. multicenter phase III study. Radiology 2005; 237: 89-98

[7] Hammerstingl R, Huppertz A, Breuer J et al. Diagnostic efficacy of gadoxetic acid (Primovist)-enhanced MRI and spiral CT for a therapeutic strategy: comparison with intraoperative and histopathologic findings in focal liver lesions. Eur Radiol 2008; 18: 457-467

[8] Vogl T], Kümmel S, Hammerstingl R et al. Liver tumors: comparison of MR imaging with Gd-EOB-DTPA and Gd-DTPA. Radiology 1996; 200: $59-67$

[9] Uggowitzer MM, Gotschuli G, Reiter H et al. Contrast-enhanced sonography of the liver. Radiologe 2005; 45: 24-33

[10] Vogl T], Bartolozzi C. Magnetic Resonance Imaging in Liver Disease: Technical Approach, Diagnostic Imaging of Liver Neoplasms, Focus on a New Superparamagnetic Contrast Agent. 2003

[11] Ba-Ssalamah A, Happel B, Kettenbach J et al. MRT of the liver. Clinical significance of nonspecific and liver-specific MRT contrast agents. Radiologe 2004; 44: 1170-1184

[12] Bittencourt LK, Hausmann D, Gasparetto EL et al. Magnetic resonance of the liver with hepato-specific contrast: initial clinical experience in Brazil. Rev Col Bras Cir 2012; 40: 237-240

[13] Neri E, Bali MA, Ba-Ssalamah A et al. ESGAR consensus statement on liver MR imaging and clinical use of liver-specific contrast agents. Eur Radiol 2016; 26: $921-931$

[14] Bauditz J, Schade T, Wermke W. Sonographic diagnosis of hilar cholangiocarcinomas by the use of contrast agents. Ultraschall in Med 2007; 28: $161-167$

[15] Bleuzen A, Huang C, Olar M et al. Diagnostic accuracy of contrast-enhanced ultrasound in focal lesions of the liver using cadence contrast pulse sequencing. Ultraschall in Med 2006; 27: 40-48

[16] Celli N, Gaiani S, Piscaglia F et al. Characterization of liver lesions by realtime contrast-enhanced ultrasonography. Eur J Gastroenterol Hepatol 2007; 19: 3-14

[17] Dietrich CF. Comments and illustrations regarding the guidelines and good clinical practice recommendations for contrast-enhanced ultrasound (CEUS) - Update 2008. Ultraschall in Med 2008; 29: 188-202

[18] Dietrich CF, Mertens JC, Braden B et al. Contrast-enhanced ultrasound of histologically proven liver hemangiomas. Hepatology 2007; 45: 1139_ 1145

[19] Dörffel Y, Wermke W. Neuroendocrine tumors: Characterization with contrast-enhanced ultrasonography. Ultraschall in Med 2008; 29: 506 514

[20] Konopke R, Bunk A, Kersting S. Contrast-enhanced ultrasonography in patients with colorectal liver metastases after chemotherapy. Ultraschall in Med 2008; 29 (Suppl. 4): S203-S209
[21] Nicolau C, Vilana R, Catalá V et al. Importance of evaluating all vascular phases on contrast-enhanced sonography in the differentiation of benign from malignant focal liver lesions. Am J Roentgenol 2006; 186: $158-167$

[22] Oldenburg A, Albrecht T. Baseline and contrast-enhanced ultrasound of the liver in tumor patients. Ultraschall in Med 2008; 29: 488-498

[23] Piscaglia F, Venturi A, Mancini M et al. Diagnostic features of real-time contrast-enhanced ultrasound in focal nodular hyperplasia of the liver. Ultraschall in Med 2010; 31: 276-282

[24] Quaia E, Calliada F, Bertolotto M et al. Characterization of focal liver lesions with contrast-specific US modes and a sulfur hexafluoride-filled microbubble contrast agent: diagnostic performance and confidence. Radiology 2004; 232: 420-430

[25] Strobel D, Kleinecke C, Hänsler ] et al. Contrast-enhanced sonography for the characterisation of hepatocellular carcinomas-correlation with histological differentiation. Ultraschall in Med 2005; 26: 270-276

[26] Xu HX, Liu G], Lu MD et al. Characterization of small focal liver lesions using real-time contrast-enhanced sonography: diagnostic performance analysis in 200 patients. J Ultrasound Med 2006; 25: 349-361

[27] Kokudo N, Hasegawa K, Akahane M et al. Evidence-based Clinical Practice Guidelines for Hepatocellular Carcinoma: The Japan Society of Hepatology 2013 update (3rd JSH-HCC Guidelines). Hepatol Res 2015; 45: $123-127$

[28] Sommer CM, Stampfl U, Kauczor HU et al. Nationale S3-Leitlinie hepatozelluläres Karzinom. Radiologe 2014; 54: 642 - 653

[29] Bolondi L, Cillo U, Colombo M et al. Position paper of the Italian Association for the Study of the Liver (AISF): The multidisciplinary clinical approach to hepatocellular carcinoma. Dig Liver Dis 2013; 45: 712 - 723

[30] Quaia E. Solid focal liver lesions indeterminate by contrast-enhanced CT or MR imaging: the added diagnostic value of contrast-enhanced ultrasound. Abdom Imaging 2012; 37: 580 - 590

[31] Quaia E, De Paoli L, Angileri R et al. Indeterminate solid hepatic lesions identified on non-diagnostic contrast-enhanced computed tomography: assessment of the additional diagnostic value of contrast-enhanced ultrasound in the non-cirrhotic liver. Eur J Radiol 2014; 83: 456-462

[32] Bernatik T, Schuler A, Kunze G et al. Benefit of Contrast-Enhanced Ultrasound (CEUS) in the Follow-Up Care of Patients with Colon Cancer: A Prospective Multicenter Study. Ultraschall in Med 2015; 36: 590-593

[33] Schellhaas B, Wildner D, Pfeifer L et al. LI-RADS-CEUS - Proposal for a Contrast-Enhanced Ultrasound Algorithm for the Diagnosis of Hepatocellular Carcinoma in High-Risk Populations. Ultraschall in Med 2016; 37: $627-634$

[34] Seitz K, Bernatik T, Strobel D et al. Contrast-enhanced ultrasound (CEUS) for the characterization of focal liver lesions in clinical practice (DEGUM Multicenter Trial): CEUS vs. MRI-a prospective comparison in 269 patients. Ultraschall in Med 2010; 31: 492-499

[35] D'Onofrio M, Crosara S, De Robertis R et al. Malignant focal liver lesions at contrast-enhanced ultrasonography and magnetic resonance with hepatospecific contrast agent. Ultrasound 2014; 22: 91 - 98

[36] Zech C], Reiser MF, Herrmann KA. Imaging of hepatocellular carcinoma by computed tomography and magnetic resonance imaging: state of the art. Dig Dis 2009; 27: 114-124

[37] Tanaka M, Kishi Y, Esaki M et al. Feasibility of Routine Application of Gadoxetic Acid-Enhanced MRI in Combination with Diffusion-Weighted MRI for the Preoperative Evaluation of Colorectal Liver Metastases. Ann Surg Oncol 2016; 23: 3991-3998

[38] Lorusso A, Quaia E, Poillucci G et al. Activity-based cost analysis of contrast-enhanced ultrasonography (CEUS) related to the diagnostic impact in focal liver lesion characterisation. Insights Imaging 2015; 6: 499-508 